\title{
Birt-Hogg-Dube syndrome presenting as multiple oncocytic parotid tumors
}

\author{
Noralane M Lindor ${ }^{1 *}$, Jan Kasperbauer ${ }^{2}$, Jean E Lewis ${ }^{3}$ and Mark Pittelkow ${ }^{4}$
}

\begin{abstract}
Mutations in FLCN cause Birt-Hogg-Dubé syndrome, an autosomal dominant disorder notable for development of cutaneous fibrofolliculomas or trichodiscomas, a variety of renal tumors, and spontaneous pneumothorax due to cystic lung changes. We present a woman referred for genetic evaluation due to bilateral parotid gland tumors, who was subsequently diagnosed with Birt-Hogg-Dubé syndrome.
\end{abstract}

Keywords: Salivary gland, Oncocytic, Warthins tumor, Oncocytosis

\section{Introduction}

BHD syndrome is an uncommon genodermatosis characterized by the presence of multiple fibrofolliculomas, trichodiscomas, and acrochordons of the skin. There is significantly increased susceptibility to renal tumors, of which about half are hybrid chromophobe/oncocytic renal cancers; about 5\% are oncocytomas. Pulmonary cysts occur in the majority of adults with BHD syndrome, leading to spontaneous pneumothorax in at least a quarter of affected individuals. A longer list of tumors have been reported rarely in BHD [1]; parotid tumors have been reported several times but have not served as the sentinel lesion bringing a patient to diagnosis.

\section{Case report}

A previously healthy 45 year old Caucasian woman had a magnetic resonance scan for persistent mild hearing loss in her right ear. The cause for the hearing loss was not identified but the magnetic resonance imaging demonstrated multiple small parotid masses: a $9 \mathrm{~mm}$ diameter peripherally enhancing/T2 hyperintense lesion in anterolateral aspect of right parotid gland, a few additional smaller T1 hypointense nonenhancing lesions in right parotid gland and additional lesions in the superficial and deep lobes of the left parotid gland (Figure 1). Fine needle aspiration biopsies revealed a mildly hypercellular collection of epithelial cells with oncocytic differentiation and associated

\footnotetext{
*Correspondence: nlindor@mayo.edu

'Department of Health Science Research, Mayo Clinic Arizona

Full list of author information is available at the end of the article
}

lymphoid aggregates; the interpretation was: suspicious for oncocytic neoplasm, favor Warthin tumor. The differential diagnosis included the spectrum of oncocytic proliferations which includes Warthin tumors, nodular oncocytic hyperplasia (nodular oncocytosis), and multiple oncocytomas of the parotid glands. The presence of lymphoid aggregates favors the diagnosis of Warthin tumors. The histologic diagnosis could not be further refined based upon the tissues obtained. Given the FNA findings and the distribution of the lesions, the plan is for observation of the parotid nodules until bothersome (size, cosmesis) and the treatment will require surgery addressing both the superficial and deep lobes of the parotid gland given the diffuse distribution of the nodules. She reported no prior history of tumors but had had several "moles" removed from her face for cosmetic reasons which were labeled only as "benign". There was no history of spontaneous pneumothorax.

The family history was notable for maternal grandfather with prostate cancer, maternal grandmother with a bladder cancer diagnosed in her 40 s and a lung cancer diagnosed in her 50s (she had smoked). A maternal uncle had a throat cancer and died at 58. The paternal family history was negative for neoplasms. Her three siblings and two children were apparently healthy.

Her physical examination was normal except for a striking number of raised, smooth, flesh-colored cutaneous papules most notable around the scalp (Figure 2) and face in a generalized distribution and around the neck accompanied by numerous acrochordons. No intraoral lesions were noted. Skin biopsies showed 


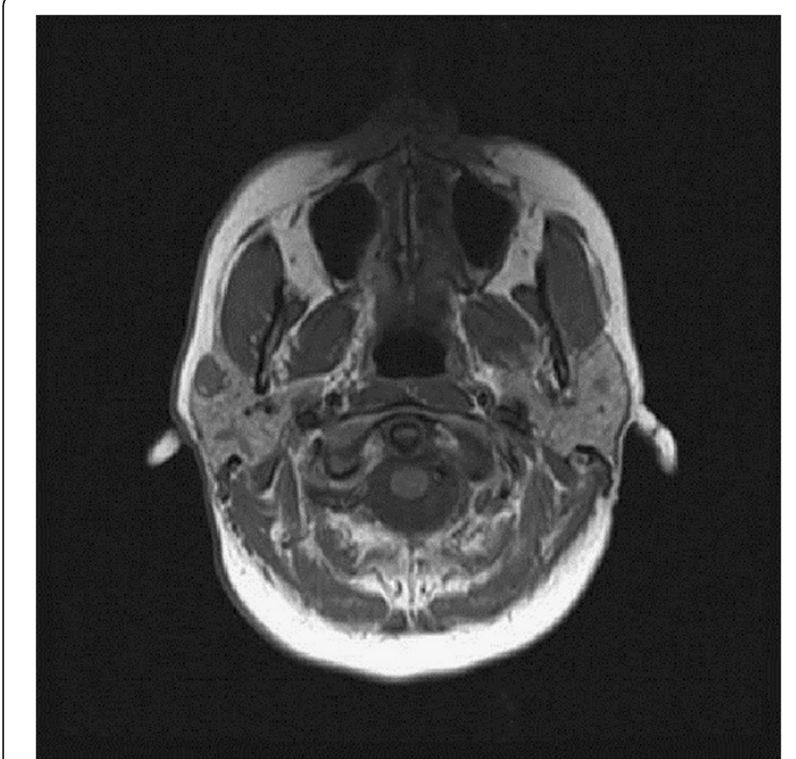

Figure 1 Axial T1 image demonstrating the largest right parotid nodule near the anterior edge of the gland with a small nodule in the posterior gland and a nodule in the left anterior parotid gland.

findings of fibrofolliculomas in the scalp lesions and acrochordons on the neck. Imaging of the kidneys by computerized tomography showed only a single small cyst, but the bases of the lungs showed extensive cystic changes. Mutation analysis of FLCN revealed a c.779 + $1 \mathrm{G}>\mathrm{T}$ mutation which has been reported previously in BHD syndrome. Tissue from the biopsy was not available for further studies such as loss of heterozygosity of $F L C N$.

\section{Discussion}

Parotid gland oncocytic lesions include a spectrum of histologic findings including nodular oncocytic hyperplasia, benign oncocytoma, and oncocytic carcinoma, and it may be reasonable to include Warthin tumor somewhere in this spectrum. Nodular oncocytic hyperplasia, if this is the correct description of the case comprises only $0.1 \%$ of parotid gland diseases [2] while $0.7 \%$ of 3,500 salivary gland tumors in another series contained benign oncocytic lesions [3]. There are a number of

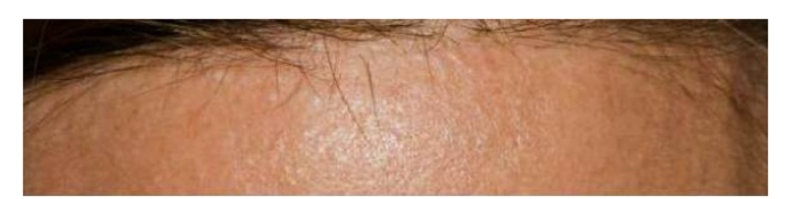

Figure 2 Inset: Whitish papules surrounding follicular orifice, especially prominent within anterior scalp hairline. individual case reports suggesting that this disorder appears to be so uncommon as to warrant single case report presentations. Warthin tumors (also called papillary cystadenoma lymphomatosum, adenolymphomas, or cystadenolymphomas) are not so rare, being the second most common tumor of salivary glands (after pleomorphic adenomas), and characteristically affect older males, and are uncommonly bilateral.

A solitary parotid oncocytoma was reported in a 56 year old man with Birt-Hogg-Dubé's syndrome [4]. In 2005, Schmidt et al., reported on 219 individuals with BHD syndrome studied at that National Cancer Institute and parotid tumors were reported in two women (ages 62 and 72) and two men (ages 20 and 39). Three of these were oncocytomas but no information about multiplicity was provided [5]. Palmirotta et al., reported on two new BHD families and one woman had a parotid pleomorphic adenoma at age 43 [6]. In 2008, Toro et al., reported on 51 families (50 were new) with 89 gene mutation carriers and in this series two individuals has parotid gland oncocytomas,at ages 20 and 39 (it is not clear if this overlapped the Schmidt study or if the same ages were coincidental) [7]. Finally, Maffé et al., reported two parotid tumors among 19 patients with suspected BirtHogg-Dubé syndrome: a Warthin parotid tumor occurred in a 59 year old male in whom no FLNC mutation was found; and of most relavance to the current report, bilateral parotid tumors not otherwise specified, occurred at ages 32 and 43 years old in one man with a documented FLNC mutation [8].

Finding an oncocytic tumor in the parotid is of interest as the renal tumors which are characteristic of BHD often contain oncocytic cells. The relevance of this similarity is obscure. Oncocytic neoplasms, in general, are thought to result from cells that accumulate abundant mitochondria in their cytoplasm to form oncocytes via oncocytic metaplasia. They account for less than $1 \%$ of salivary gland tumors and can occur in other organs including kidneys, thyroid and parathyroid glands [9].

Molecular studies of Warthin tumors of the salivary glands indicate a recurrent $t(11 ; 19)$ translocation with associated CRTC1-MAML2 fusion oncogene in a subset of Warthin tumors [10]. We were not able to study this biopsy for this finding. The chromosomal location of FLCN is $17 \mathrm{p} 11.2$ so no direct link to this translocated region is evident. Comparative genomic hybridization studies of salivary tumors did show some tumors with deletions involving 17p11.2 region in apparently 6 of 15 Warthin tumors but details in that publication were insufficient to determine if FLCN was deleted or not [11].

The function of $F L C N$ is still a matter of investigation. Baba et al., [12] have studied the protein and stated that "the interaction of FLCN with FNIP1 and AMPK suggests a possible role for FLCN and FNIP1 in the 


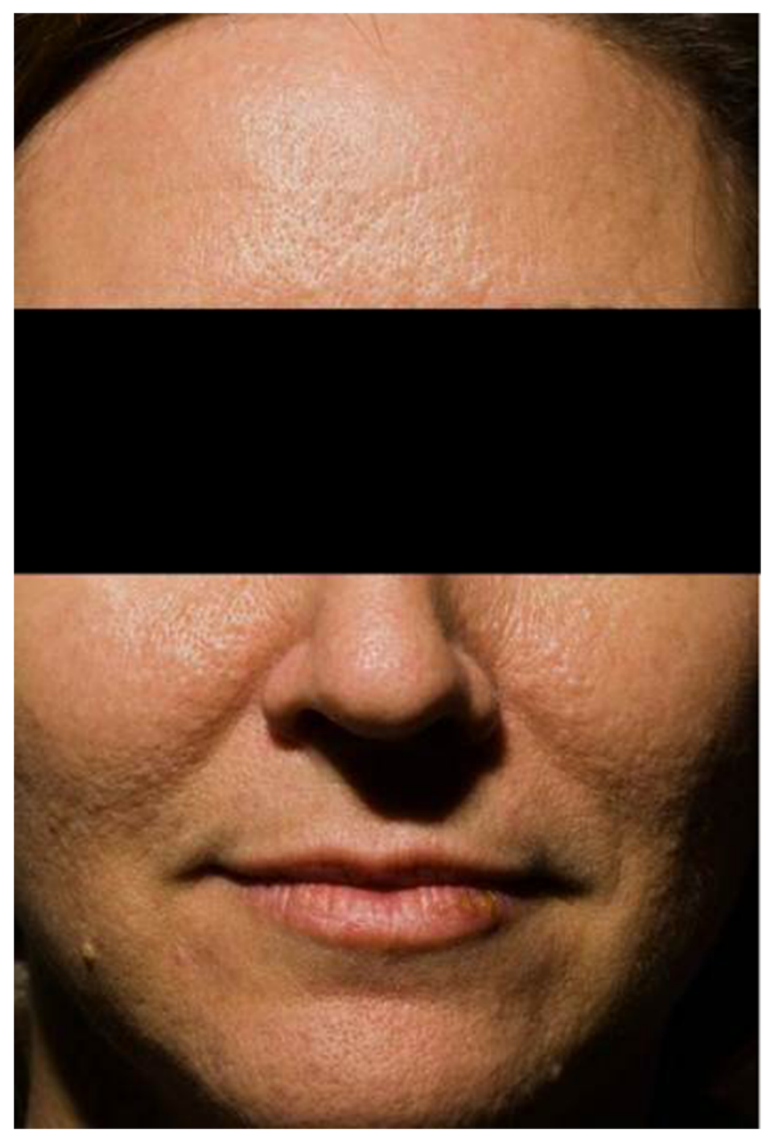

Figure 3 Face and forehead. Follicle-based, papular appearance of cheeks and forehead.

nutrient/energy-sensing pathways involving AMPK and mTOR and may provide a molecular mechanism for the BHD phenotype". No direct link to mitochondrial dysfunction has been proposed yet for this tumor suppressor gene but the oncocytic nature of the tumors in both kidney and now parotid gland raise this as a possibility.

Around half of BHD families recognized to date have a mutation in hot spot involving deletion (c.1285delC) or duplication (c.1285dupC) of a C nucleotide in the polycytosine tract in exon 11 of FLCN [5]. The mutation found in this patient is not in this area but has been reported previously in six individuals from two families [as IVS7 + 1] [7] and has not been reported since, per the Leiden Open Variome Database update of 2011 [https://grenada.lumc.nl/LOVD2/shared1/variants. php?select_db=FLCN\&action=view_unique]. The major manifestation in these families generally appeared typical of BHD syndrome with renal tumors in five, lung cysts in six, documented fibrofolliculomas in five. Also reported was an angiofibroma, dermatofibrosarcoma protuberans, cutaneous leiomyosarcoma, and trichodiscoma. No parotid tumors were reported. Combined with the current case report, these families might be perceived as having more diverse dermatological findings than other BHD families, raising the possibility of some genotype-phenotype interaction.

Based upon the DNA diagnosis, the patient was counseled regarding autosomal dominant inheritance of this syndrome and the implications for multiple relatives, and was provided screening recommendations) for typical BHD syndrome with regard to renal and pulmonary complications, and she will remain under closer surveillance for changes in the parotid tumors and for additional dermatologic findings [13].

\section{Consent}

Written consent for use of patient photographs were obtained.

\section{Competing interests}

All authors' have declared that they have no competing interest.

\section{Author contributions}

All authors contributed to clinical diagnosis and review of the manuscript. All authors read and approved the final manuscript.

\section{Acknowledgements}

We thank Danielle Wright for preparation and submission of this manuscript. 


\section{Author details}

'Department of Health Science Research, Mayo Clinic Arizona ${ }^{2}$ Department of Otolaryngology. ${ }^{3}$ Department of Laboratory Medicine and Pathology. ${ }^{4}$ Department of Dermatology, Mayo Clinic Rochester.

Received: 5 April 2012 Accepted: 10 September 2012 Published: 10 October 2012

\section{References}

1. Reese E, Sluzevich J, Kluijt I, Teertstra HJ, De Jong D, Horenblas S, Ryu J: Birt-Hogg-Dube Syndrome. 2009

2. Strassburger S, Hyckel P, Kosmehl H: Multifocal oncocytic adenomatous hyperplasia of the parotid gland. A case report. Int I Oral Maxillofac Surg 1999, 28(6):457-458

3. Palmer TJ, Gleeson MJ, Eveson JW, Cawson RA: Oncocytic adenomas and oncocytic hyperplasia of salivary glands: a clinicopathological study of 26 cases. Histopathology 1990, 16(5):487-493.

4. Liu V, Kwan T, Page EH: Parotid oncocytoma in the Birt-Hogg-Dube syndrome. J Am Acad Dermatol 2000, 43(6):1120-1122.

5. Schmidt LS, Nickerson ML, Warren MB, et al: Germline BHD-mutation spectrum and phenotype analysis of a large cohort of families with Birt-Hogg-Dube syndrome. Am J Hum Genet 2005, 76(6):1023-1033.

6. Palmirotta R, Donati P, Savonarola A, Cota C, Ferroni P, Guadagni F: Birt-Hogg-Dube (BHD) syndrome: report of two novel germline mutations in the folliculin (FLCN) gene. Eur J Dermatol 2008 18(4):382-386

7. Toro JR, Wei MH, Glenn GM, et al: BHD mutations, clinical and molecular genetic investigations of Birt-Hogg-Dube syndrome: a new series of 50 families and a review of published reports. J Med Genet 2008, 45(6):321-331.

8. Maffe A, Toschi B, Circo G, et al: Constitutional FLCN mutations in patients with suspected Birt-Hogg-Dube syndrome ascertained for noncutaneous manifestations. Clin Genet 2011, 79(4):345-354.

9. Shellenberger TD, Williams MD, Clayman GL, Kumar AJ: Parotid gland oncocytosis: CT findings with histopathologic correlation. AJNR Am J Neuroradiol 2008, 29(4):734-736.

10. O'Neill ID: New insights into the nature of Warthin's tumour. J Oral Pathol Med 2009, 38(1):145-149.

11. Giefing M, Wierzbicka M, Rydzanicz M, Cegla R, Kujawski M, Szyfter K Chromosomal gains and losses indicate oncogene and tumor suppressor gene candidates in salivary gland tumors. Neoplasma 2008, 55(1):55-60.

12. Baba M, Hong SB, Sharma N, et al: Folliculin encoded by the BHD gene interacts with a binding protein, FNIP1, and AMPK, and is involved in AMPK and mTOR signaling. Proc Natl Acad Sci USA 2006, 103(42):15552-15557.

13. Riegert-Johnson D, Boardman L, Hefferon T, et al: Cancer Syndromes. 2009. http://www.ncbi.nlm.nih.gov/pubmed/21249754.

doi:10.1186/1897-4287-10-13

Cite this article as: Lindor et al:: Birt-Hogg-Dube syndrome presenting as multiple oncocytic parotid tumors. Hereditary Cancer in Clinical Practice 2012 10:13

\section{Submit your next manuscript to BioMed Central and take full advantage of:}

- Convenient online submission

- Thorough peer review

- No space constraints or color figure charges

- Immediate publication on acceptance

- Inclusion in PubMed, CAS, Scopus and Google Scholar

- Research which is freely available for redistribution 